\title{
State-to-state Mode Specificity: Energy Sequestration and Flow Gated by Transition State
}

\author{
Bin Zhao, ${ }^{1}$ Zhigang Sun, ${ }^{2}$ and Hua Guo ${ }^{1, *}$ \\ ${ }^{1}$ Department of Chemistry and Chemical Biology, University of New Mexico, Albuquerque, New Mexico \\ 87131, USA \\ ${ }^{2}$ State Key Laboratory of Molecular Reaction Dynamics and Center for Theoretical and Computational \\ Chemistry, Dalian Institute of Chemical Physics, Chinese Academy of Sciences, Dalian 116023, China
}

\footnotetext{
* Corresponding author: hguo@unm.edu
} 
S1. Theory

\section{S1.1. Hamiltonian and Wavefunctions}

Transition-state wave packet (TSWP) calculations at the state-to-state level were carried out in both the diatom-diatom and atom-triatom Jacobi coordinates. As shown in Fig. S1, Jacobi coordinates for the $\mathrm{H}_{2}+\mathrm{OH}$ and $\mathrm{H}+\mathrm{H}_{2} \mathrm{O}$ arrangements are denoted as $\left(R, r_{1}, r_{2}, \theta_{1}, \theta_{2}, \varphi\right)$ and $\left(R^{\prime}, r_{1}^{\prime}, r_{2}^{\prime}, \theta_{1}^{\prime}, \theta_{2}^{\prime}, \varphi^{\prime}\right)$, respectively. The $\boldsymbol{R}$ and $\boldsymbol{R}^{\prime}$ vectors are defined as the $z$-axis of the body-fixed (BF) frame for the $\mathrm{H}_{2}+\mathrm{OH}$ and $\mathrm{H}+\mathrm{H}_{2} \mathrm{O}$ arrangements, respectively, and the vector $\boldsymbol{r}_{1}$ and $\boldsymbol{r}_{1}^{\prime}$ lies in the corresponding $x$-z planes, respectively.

The Hamiltonians for both the $\mathrm{H}_{2}+\mathrm{OH}$ and $\mathrm{H}+\mathrm{H}_{2} \mathrm{O}$ arrangements have formally the same form $(\hbar=1$ hereafter $){ }^{1}$

$$
\hat{H}=-\frac{1}{2 \mu} \frac{\partial^{2}}{\partial R^{2}}+\frac{\left(\hat{J}-\hat{j}_{12}\right)^{2}}{2 \mu R^{2}}+\sum_{i=1}^{2}\left[\hat{h}_{i}\left(r_{i}\right)+\frac{\hat{j}_{i}^{2}}{2 \mu_{i} r_{i}^{2}}\right]+\left[V\left(R, r_{1}, r_{2}, \theta_{1}, \theta_{2}, \varphi\right)-\sum_{i=1}^{2} V_{i}\left(r_{i}\right)\right] .
$$

In the $\mathrm{H}_{2}+\mathrm{OH}$ Hamiltonian, $\mu_{1}$ and $\mu_{2}$ are diatomic reduced masses for $\mathrm{H}_{2}$ and $\mathrm{OH}, \mu$

is the reduced mass between the COMs of $\mathrm{H}_{2}$ and $\mathrm{OH} . \hat{j}_{1}$ and $\hat{j}_{2}$ are the rotational angular momentum operators of $\mathrm{H}_{2}$ and $\mathrm{OH}$, respectively, and they are coupled to form $\hat{j}_{12}$. In the $\mathrm{H}+\mathrm{H}_{2} \mathrm{O}$ Hamiltonian, $\mu^{\prime}$ is the reduced mass between $\mathrm{H}$ and the COM of $\mathrm{H}_{2} \mathrm{O}, \mu_{1}^{\prime}$ is the reduced mass between $\mathrm{H}$ and the $\mathrm{COM}$ of $\mathrm{OH}$, and $\mu_{2}^{\prime}$ is the reduced mass of $\mathrm{OH} . \hat{j}_{12}^{\prime}$ is the total angular momentum operator of $\mathrm{H}_{2} \mathrm{O}, \hat{j}_{2}^{\prime}$ is the rotational angular momentum operator of $\mathrm{OH}$, and $\hat{j}_{1}^{\prime}=\hat{j}_{12}^{\prime}-\hat{j}_{2}^{\prime}$ is the orbital angular momentum 
operator of $\mathrm{H}$ relative to $\mathrm{OH}$. For both cases, $J$ is the conserved total angular momentum operator of the system and chosen to be 0 in this study. The reference vibrational Hamiltonians $\hat{h}_{i}\left(r_{i}\right)$ are defined as

$$
\hat{h}_{i}\left(r_{i}\right)=-\frac{1}{2 \mu_{i}} \frac{\partial^{2}}{\partial r_{i}^{2}}+V_{i}\left(r_{i}\right), \quad(i=1,2),
$$

and $V_{i}\left(r_{i}\right)$ are the one-dimensional (1D) reference potentials. The wavefunction is expanded in terms of BF bases,

$$
\Psi^{J M \varepsilon}\left(\boldsymbol{R}, \boldsymbol{r}_{1}, \boldsymbol{r}_{2}, t\right)=\sum_{n, \nu, j, K} F_{n v j K}^{J M \varepsilon}(t) u_{n}^{v_{1}}(R) \phi_{v_{1}}^{1}\left(r_{1}\right) \phi_{v_{2}}^{2}\left(r_{2}\right) y_{j K}^{J M \varepsilon}\left(\hat{R}, \hat{r}_{1}, \hat{r}_{2}\right)
$$

where $n$ is the index of the translational basis $u_{n}^{v_{1}}(R)$, which is defined as

$$
u_{n}^{v_{1}}(R)=\left\{\begin{array}{l}
\sqrt{\frac{2}{R_{3}-R_{1}}} \sin \frac{n \pi R}{R_{3}-R_{1}}, v_{1} \leq v_{a s y} \\
\sqrt{\frac{2}{R_{2}-R_{1}}} \sin \frac{n \pi R}{R_{2}-R_{1}}, v_{1}>v_{a s y}
\end{array},\right.
$$

where $R_{2}$ separates the $R$ coordinate range $\left(R_{1}, R_{3}\right)$ into the asymptotic and interaction regions in an $L$-shaped scheme, ${ }^{9}$ and different translational bases are used in the two regions. $v_{a s y}$ is chosen to be the number of energetically open vibrational channels plus one or two closed channels. $\phi_{\nu_{i}}^{i}\left(r_{i}\right)$ are the eigenfunctions of the reference vibrational Hamiltonians $\hat{h}_{i}\left(r_{i}\right) . v$ is a composite index and it denotes the two vibrational states, $v_{1}$ and $v_{2} . M$ is the projection of $J$ in the space-fixed (SF) framework and $\varepsilon$ is the parity. The composite index $j$ denotes $\left(j_{1}, j_{2}, j_{12}\right), \varepsilon$ is the parity of the system defined as 
$\varepsilon=(-1)^{j_{1}+j_{2}+l}$ with $l$ being the quantum number of the orbital angular momentum operator $\hat{l}=\hat{J}-\hat{j}_{12}$. The parity-adapted BF rotational basis $y_{j K}^{J M \varepsilon}\left(\hat{R}, \hat{r}_{1}, \hat{r}_{2}\right)$ is given as

$$
\begin{aligned}
y_{j K}^{J M \varepsilon}\left(\hat{R}, \hat{r}_{1}, \hat{r}_{2}\right)= & \left(1+\delta_{K 0}\right)^{-1 / 2} \sqrt{\frac{2 J+1}{8 \pi}}\left[D_{K, M}^{J *}(\alpha, \beta, \gamma) Y_{j_{1} j_{2}}^{j_{12} K}\left(\theta_{1}, \theta_{2}, \varphi\right)\right. \\
& \left.+\varepsilon(-1)^{j_{1}+j_{2}+j_{j_{2}}+J} D_{-K, M}^{J^{*}}(\alpha, \beta, \gamma) Y_{j_{1} j_{2}}^{j_{1}-K}\left(\theta_{1}, \theta_{2}, \varphi\right)\right],
\end{aligned}
$$

where $D_{K, M}^{J *}(\alpha, \beta, \gamma)$ is the Wigner rotation matrix ${ }^{2}$ that rotates the $\mathrm{SF}$ frame to $\mathrm{BF}$ frame by three Euler angles $(\alpha, \beta, \gamma)$. The restriction that $\varepsilon(-1)^{j_{1}+j_{2}+j_{12}+J}=1$ for $K=0$ partitions the whole rotational basis set into even and odd parities. It should be noted here that the definitions of $Y_{j_{1} j_{2}}^{j_{1} K}\left(\theta_{1}, \theta_{2}, \varphi\right)$ are different for the $\mathrm{H}_{2}+\mathrm{OH}$ and $\mathrm{H}+\mathrm{H}_{2} \mathrm{O}$ arrangements. The $\mathrm{H}_{2}+\mathrm{OH}$ rotational basis $Y_{j_{1} j_{2}}^{j_{1} K}\left(\theta_{1}, \theta_{2}, \varphi\right)$ is given by

$$
Y_{j_{1} j_{2}}^{j_{1} K}\left(\theta_{1}, \theta_{2}, \varphi\right)=\sum_{m_{1}}\left\langle j_{1} m_{1} j_{2} K-m_{1} \mid j_{12} K\right\rangle y_{j_{1} m_{1}}\left(\theta_{1}, 0\right) y_{j_{2} K-m_{1}}\left(\theta_{2}, \varphi\right)
$$

where $y_{j m}$ are spherical harmonics, and $\left\langle j_{1} m_{1} j_{2} K-m_{1} \mid j_{12} K\right\rangle$ are the Clebsch-Gordan coefficients. ${ }^{2}$ The $\mathrm{H}+\mathrm{H}_{2} \mathrm{O}$ rotational basis $Y_{j_{1}^{\prime} j_{2}^{\prime}}^{j_{1}^{\prime} K^{\prime}}\left(\theta_{1}^{\prime}, \theta_{2}^{\prime}, \varphi^{\prime}\right)$ is on the other hand given by

$$
Y_{j_{1}^{\prime} j_{2}^{\prime}}^{j_{1}^{\prime} K^{\prime}}\left(\theta_{1}^{\prime}, \theta_{2}^{\prime}, \varphi^{\prime}\right)=\sum_{m} D_{K^{\prime} m}^{j_{1}^{\prime}, *}\left(0, \theta_{1}^{\prime}, \varphi^{\prime}\right) \sqrt{\frac{2 j_{1}^{\prime}+1}{4 \pi}}\left\langle j_{2}^{\prime} m j_{1}^{\prime} 0 \mid j_{12}^{\prime} m\right\rangle y_{j_{2} m}\left(\theta_{2}^{\prime}, 0\right),
$$

where the Wigner rotation matrix $D_{K^{\prime} m}^{j_{1}^{\prime} *}\left(0, \theta_{1}^{\prime}, \varphi^{\prime}\right)$ rotates the $\mathrm{BF}$ frame to the MF frame (the $\mathrm{z}$ axis of this frame lies along vector $\left.\boldsymbol{r}_{1}^{\prime}\right)$ by the Euler angles $\left(0, \theta_{1}^{\prime}, \varphi^{\prime}\right)$. 


\section{S1.2. Thermal Flux Operator}

In the TSWP method, the definition of thermal flux operator, $\hat{F}_{T}=e^{-\hat{H} / 2 k_{B} T} \hat{F} e^{-\hat{H} / 2 k_{B} T}$, is based on flux operator $\hat{F}=i[\hat{H}, h]$ with $h$ as the Heaviside

function. ${ }^{3}$ Because the singular character of the flux operator $\hat{F}$ and the divergence of its eigenvalues with respect to the largest momentum supported on the grid, an accurate representation of the flux operator eigenfunctions is numerically difficult. The Boltzmann operators in $\hat{F}_{T}$ remove the singularity and render the thermal flux eigenstates smooth in the coordinate representation. As a result, higher numerical accuracy can be achieved.

The thermal flux operator can be expanded in the spectral representation, ${ }^{4}$

$$
\hat{F}_{T}=\sum_{n} f_{T}^{n}\left|f_{T}^{n}\right\rangle\left\langle f_{T}^{n}\right|
$$

where $f_{T}^{n}$ and $\left|f_{T}^{n}\right\rangle$ are the eigenvalues and eigenfunctions of the low-rank thermal flux operator, respectively. The real eigenvalues $f_{T}^{n}$ come in pairs with the same absolute value but different signs, ${ }^{5}$ and the corresponding eigenstates $\left|f_{T}^{n}\right\rangle$ come in complex conjugate pairs and they can be interpreted as the ro-vibrational wavefunctions of the activated complex in the transition-state region.

The eigenstates $\left|f_{T}^{n}\right\rangle$ are defined on a dividing surface near the transition-state, which is defined on the $R^{\prime}-r_{1}^{\prime}$ plane of the product arrangements as follows:

$$
q=\left(r_{1}^{\prime}-r_{10}^{\prime}\right)-\left(R^{\prime}-R_{0}^{\prime}\right) \tan \chi=0,
$$

where $\chi$ is the angle of the line to the $R_{0}^{\prime}$ axis and $\left(R_{0}^{\prime}, r_{10}^{\prime}\right)$ is a point in the $R^{\prime}-r_{1}^{\prime}$ 
plane. The flux operator can then be expressed as

$$
\hat{F}=i[\hat{H}, h(q)]=\hat{F}_{R^{\prime}}+\hat{F}_{r_{1}^{\prime}}
$$

where

$$
\begin{aligned}
& \hat{F}_{R^{\prime}}=\frac{i}{2 \mu^{\prime}} \tan \chi\left[\frac{\partial}{\partial R^{\prime}} \delta(q)+\delta(q) \frac{\partial}{\partial R^{\prime}}\right], \\
& \hat{F}_{r_{1}^{\prime}}=-\frac{i}{2 \mu_{1}^{\prime}}\left[\frac{\partial}{\partial r_{1}^{\prime}} \delta(q)+\delta(q) \frac{\partial}{\partial r_{1}^{\prime}}\right],
\end{aligned}
$$

are the fluxes in the $R^{\prime}$ and $r_{1}^{\prime}$ coordinates, respectively.

\section{S1.3. Coordinate Transformation}

TSWPs were calculated in the $\mathrm{H}+\mathrm{H}_{2} \mathrm{O}$ Jacobi coordinate and their propagation into product asymptote is straightforward, but the propagation into reactant asymptote requires the transformation of the TSWPs to the $\mathrm{H}_{2}+\mathrm{OH}$ Jacobi coordinates. As discussed in detail previously, ${ }^{6,7}$ the transformation only needs to be carried out in five dimensions, with the $r_{2}$ and $r_{2}^{\prime}$ coordinates using the same basis functions. Briefly, the transformation is divided into two steps: first, TSWPs initially prepared in the $\mathrm{H}+\mathrm{H}_{2} \mathrm{O}$ coordinates are interpolated on the $\mathrm{H}_{2}+\mathrm{OH}$ coordinate grid points with the corresponding basis functions, then they are transformed to the basis representation by a collocation method along the $r_{1}^{\prime}$ coordinate and by transformation matrices in other coordinates. 
S1.4. Stretching wavefunctions of $\mathrm{H}_{2} \mathrm{O}$ product in the local-mode picture

To rationalize the different stretching state populations of the $\mathrm{H}_{2} \mathrm{O}$ product, one requires the invocation of the local-mode picture. The normal-mode wavefunctions $\left(\left|v_{s} v_{b} v_{a}\right\rangle\right)$ can be expanded in the form of linear combinations of the symmetrized localmode counterparts $\left(\left|(n m)^{ \pm}\right\rangle\left|v_{b}\right\rangle\right)$,

$$
\left|v_{s} v_{b} v_{a}\right\rangle=\sum C_{n m}\left|(n m)^{ \pm}\right\rangle\left|v_{b}\right\rangle
$$

where $C_{n m}$ are the expansion coefficients and $\left|(n m)^{ \pm}\right\rangle$are the symmetrized local-mode bases, which are defined as follows: ${ }^{8}$

$$
\begin{aligned}
& \left|(n m)^{ \pm}\right\rangle=1 / \sqrt{2}(|n m\rangle \pm|m n\rangle), \quad n<m, \\
& \left|(n m)^{ \pm}\right\rangle=|n m\rangle, \quad n=m,
\end{aligned}
$$

where $|n m\rangle$ denote the local-mode wavefunctions with $n$ and $m$ quanta in the two $\mathrm{OH}$ stretching modes, respectively, and it is defined as follows:

$$
|n m\rangle=\phi_{n}^{2}\left(b_{1}\right) \phi_{m}^{2}\left(b_{2}\right)
$$

where $\phi_{n}^{2}$ is the eigenfunction of the reference vibrational Hamiltonian $\hat{h}_{2}\left(r_{2}\right), b_{1}$ and $b_{2}$ are the bond lengths of the two OH bonds, respectively, as shown in Fig. S2.

Before the normal-mode wavefunction $\left|v_{s} v_{b} v_{a}\right\rangle$ could be expanded in the 
symmetrized local-mode bases, $\left|v_{s} v_{b} v_{a}\right\rangle$ is required to be transformed from the original Jacobi coordinates $\left(r_{1}^{\prime}, r_{2}^{\prime}, \theta_{2}^{\prime}\right)$ to valence coordinates $\left(b_{1}, b_{2}, a\right)$ with $b_{1} / r_{1}^{\prime}$ as the Jacobian factor for the transformation. Finally, the square of the weights $\left|C_{n m}\right|^{2}$ are readily obtained by projecting the transformed normal-mode wavefunctions onto the local-mode bases $\left(\left|(n m)^{ \pm}\right\rangle\right)$with the modulus square of the remaining degree of freedom $(a)$.

\section{S2. Numerical Details}

An $L$-shaped scheme ${ }^{9}$ is adopted to define the asymptotic and interaction regions to reduce grid size. In this scheme, the first $N_{R}^{1}$ out of $N_{R}^{2}$ grid points along the $R$ coordinates are used to define the interaction region, and the remaining grid points are to define the asymptotic region. For the $r_{1}$ coordinate, $N_{r_{1}}^{1}$ and $N_{r_{1}}^{2}$ PODVR basis functions ${ }^{10}$ are used for the interaction and asymptotic regions, respectively. The parameters used in the calculations are listed in Table S-I.

Thermal flux operator is defined in the $\mathrm{H}+\mathrm{H}_{2} \mathrm{O}$ Jacobi coordinates, and the corresponding eigenstates are obtained by using the ARPACK/PARPACK packages with implicitly restarted Arnoldi method, ${ }^{11}$ and the first $N_{T}$ pairs of thermal flux eigenstates are efficiently obtained by iteratively applying the thermal flux operator on to the Krylov vectors. The imaginary time propagation in the thermal flux operator uses a second-order split operator propagator, ${ }^{12}$ which is also adopted in the following real time propagation of thermal flux eigenstates into the reactant and product asymptotic regions. 


\section{S3. Additional results}

\section{S3.1 Convergence}

In Fig. S3, the accuracy of the state-to-state reaction probabilities is checked by comparing the sum of state-to-state reaction probabilities with results obtained by both the initial state-specific wave packet (ISSWP) method and TSWP method with only propagation towards the reactant asymptote. The TSWP results are multiplied by a factor of two to account for the two equivalent product channels of the reaction. It is seen that all the results are in very good agreement, which supports the convergence of the calcualtions.

\section{S3.2 Influence of Reactant Rotational Excitation}

The spectator character of $\mathrm{OH}$ reactant is further manifested in the final rotational state distribution of the $\mathrm{H}_{2} \mathrm{O}$ product, as shown in Fig. S4. Selected at three collision energies of $0.3,0.4$, and $0.5 \mathrm{eV}$, the two $\mathrm{OH}$ vibrational states produce almost identical distribution of $\mathrm{H}_{2} \mathrm{O}$ rotational state populations. Here, the rotational numbers of the asymmetric rotor are lumped together and no assignment was attempted. This is rationalized by noting the fact that $\mathrm{H}_{2} \mathrm{O}$ rotational state distribution is totally controlled by the transition state. The spectator $\mathrm{OH}$ reactant has no coupling to reaction coordinate, and as a result the vibration of $\mathrm{OH}$ has no ability to exert any impact on the final rotational state distribution, thus confirming the "loss of memory" effect. 
S3-4. Complete weights of symmetrized local-mode bases in several low-lying $\mathrm{H}_{2} \mathrm{O}$ normal-mode wavefunctions

The complete weights of symmetrized local-mode bases in the normal mode wavefunction of $\mathrm{H}_{2} \mathrm{O}$ product are listed in Table S-II for reference. 


\section{References:}

1. B. Zhao, Z. Sun and H. Guo, J. Chem. Phys. 141 (15), 154112 (2014).

2. R. N. Zare, Angular Momentum: Understanding Spatial Aspects in Chemistry and Physics (Wiley-Interscience, New York, 1988).

3. T. P. Park and J. C. Light, J. Chem. Phys. 88, 4897-4912 (1988).

4. F. Matzkies and U. Manthe, J. Chem. Phys. 106, 2646-2653 (1997).

5. U. Manthe, J. Chem. Phys. 102, 9205-9213 (1995).

6. M. T. Cvitaš and S. C. Althorpe, J. Chem. Phys. 134, 024309 (2011).

7. S. Liu, C. Xiao, T. Wang, J. Chen, T. Yang, X. Xu, D. H. Zhang and X. Yang, Faraday Disc. 157, 101-111 (2012).

8. M. S. Child and L. Halonen, Adv. Chem. Phys. 57, 1-58 (1984).

9. D. H. Zhang and J. Z. H. Zhang, J. Chem. Phys. 99, 5615-5618 (1993).

10. J. C. Light and T. Carrington Jr., Adv. Chem. Phys. 114, 263-310 (2000).

11. R. B. Lehoucq, D. C. Sorensen and C. Yang, ARPACK User Guide: Solution of Large Scale Eigenvalue Problems by Implicitly Restarted Arnoldi Methods. (SIAM, Philadelphia, PA, 1998).

12. M. D. Feit, J. A. Fleck Jr. and A. Steiger, J. Comput. Phys. 47, 412-433 (1982). 
Table S-I. Parameters used in the calculations

\begin{tabular}{|c|c|c|c|}
\hline \multicolumn{4}{|c|}{ Thermal flux operator } \\
\hline \multicolumn{2}{|c|}{$\chi$ (rad.) } & \multicolumn{2}{|c|}{0.08} \\
\hline \multicolumn{2}{|c|}{$R_{0}^{\prime}$} & \multicolumn{2}{|c|}{2.60} \\
\hline \multicolumn{2}{|c|}{$r_{10}^{\prime}$} & \multicolumn{2}{|c|}{2.50} \\
\hline \multicolumn{2}{|c|}{$T(K)$} & \multicolumn{2}{|c|}{4000.0} \\
\hline \multicolumn{2}{|c|}{ No. of pairs } & \multicolumn{2}{|c|}{200} \\
\hline \multicolumn{2}{|c|}{ Parameters for Reactant Coordinate } & \multicolumn{2}{|c|}{ Parameters for Product Coordinate } \\
\hline $\begin{array}{l}\text { Total time/time step } \\
\text { (a.u.) }\end{array}$ & $8000 / 10$ & $\begin{array}{l}\text { Total time/time step } \\
\text { (a.u.) }\end{array}$ & $6000 / 10$ \\
\hline Analyzing surface & $R_{\infty}=11.0$ & Analyzing surface & $R_{\infty}^{\prime}=10.0$ \\
\hline$R$ & $\begin{array}{c}R \in(1.7,14.0), \\
N_{R}^{1}=32, N_{R}^{2}=112\end{array}$ & $R^{\prime}$ & $\begin{array}{c}R^{\prime} \in(1.2,14.0), \\
N_{R}^{1}=48, N_{R}^{2}=120\end{array}$ \\
\hline$r_{1}$ & $\begin{array}{c}r_{1} \in(0.7,5.0) \\
N_{r_{1}}^{1}=32, N_{r_{1}}^{2}=8\end{array}$ & $r_{1}^{\prime}$ & $\begin{array}{c}r_{1}^{\prime} \in(0.7,5.0) \\
N_{r_{1}}^{1}=28, N_{r_{1}}^{2}=8\end{array}$ \\
\hline$r_{2}$ & $\begin{array}{c}r_{2} \in(0.7,5.0) \\
N_{r_{2}}=4\end{array}$ & $r_{2}^{\prime}$ & $\begin{array}{c}r_{2}^{\prime} \in(0.7,5.0) \\
N_{r_{2}}=4\end{array}$ \\
\hline$j_{1}$ & $j_{1} \in(0,38)$ & $j_{2}^{\prime}$ & $j_{2}^{\prime} \in(0,20)$ \\
\hline$j_{2}$ & $j_{2} \in(0,20)$ & $j_{1}^{\prime}$ & $j_{1}^{\prime} \in(0,28)$ \\
\hline
\end{tabular}


Table S-II. Complete weights of symmetrized local-mode bases in several low-lying $\mathrm{H}_{2} \mathrm{O}$ normal-mode wavefunctions

\begin{tabular}{c||c|c|c|c|c|c}
\hline \multicolumn{1}{c||}{$\left(v_{\mathrm{s}} v_{\mathrm{b}} v_{\mathrm{a}}\right)$} & \multicolumn{7}{c}{$\left|\mathrm{C}_{\mathrm{nm}}\right|^{2}(\%)$} \\
\cline { 2 - 7 } & $\mid(00)^{+}>$ & $\mid(10)^{+}>$ & $\mid(10)^{-}>$ & $\mid(20)^{+}>$ & $\mid(20)^{-}>$ & $\mid(11)^{+}>$ \\
\hline \hline$(000)$ & $\mathbf{9 9 . 7 4}$ & 0.25 & 0.00 & 0.00 & 0.00 & 0.00 \\
\hline$(100)$ & 0.76 & $\mathbf{9 8 . 2 8}$ & 0.42 & 0.28 & 0.00 & 0.26 \\
\hline$(001)$ & 0.00 & 0.41 & $\mathbf{9 9 . 2 7}$ & 0.00 & 0.30 & 0.00 \\
\hline$(200)$ & 0.02 & 1.46 & 0.06 & $\mathbf{7 6 . 8 2}$ & 4.94 & 15.83 \\
\hline$(101)$ & 0.00 & 0.07 & 1.08 & 3.48 & $\mathbf{9 3 . 1 2}$ & 1.57 \\
\hline$(002)$ & 0.00 & 0.13 & 0.00 & 18.11 & 0.13 & $\mathbf{8 1 . 1 7}$ \\
\hline
\end{tabular}




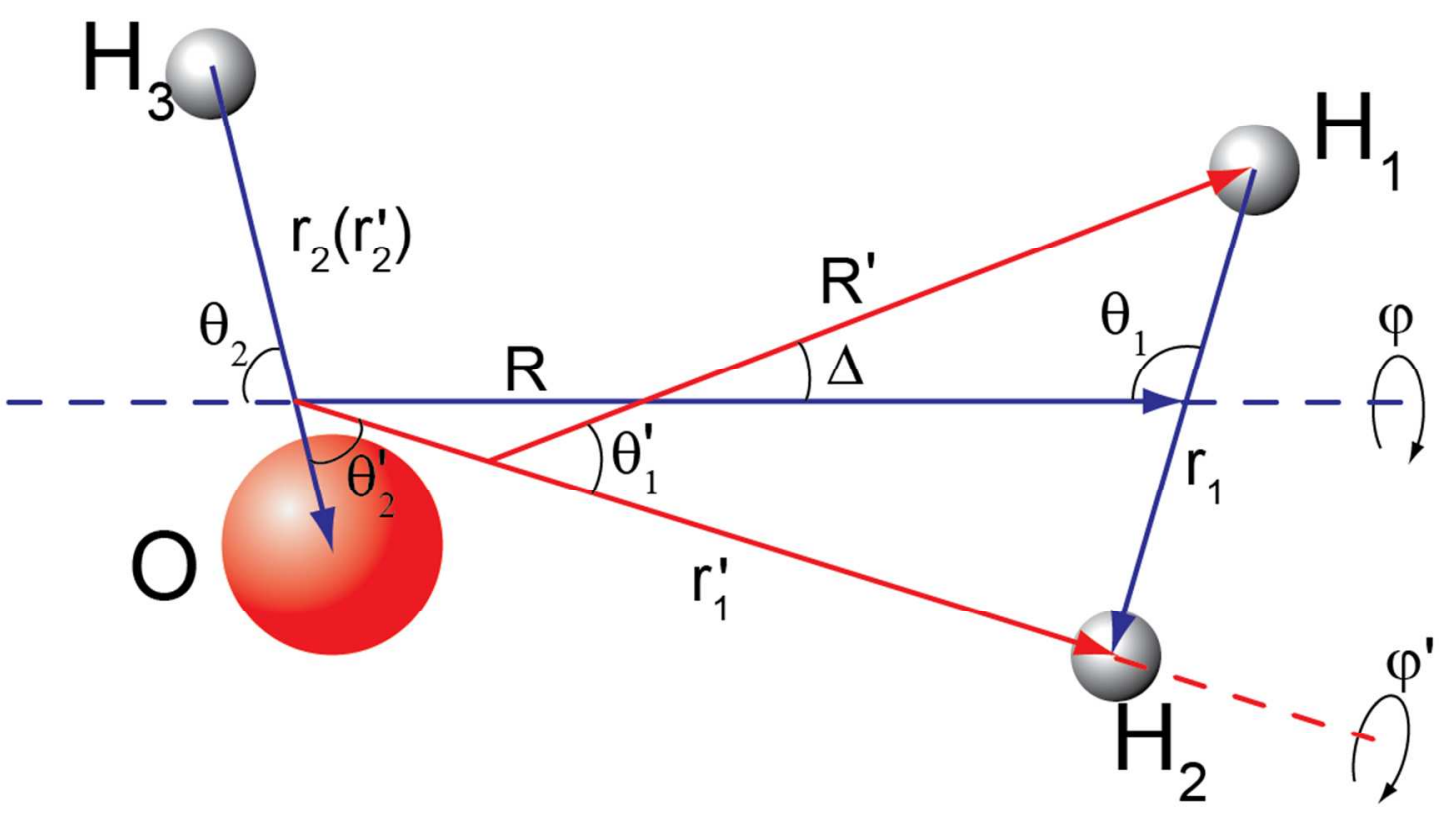

Fig. S1. The $\mathrm{H}_{2}+\mathrm{OH}$ Jacobi coordinates $\left(R, r_{1}, r_{2}, \theta_{1}, \theta_{2}, \varphi\right)$ and the $\mathrm{H}+\mathrm{H}_{2} \mathrm{O}$ Jacobi coordinates $\left(R^{\prime}, r_{1}^{\prime}, r_{2}^{\prime}, \theta_{1}^{\prime}, \theta_{2}^{\prime}, \varphi^{\prime}\right)$ for the reaction of $\mathrm{H}_{2}+\mathrm{OH} \rightarrow \mathrm{H}+\mathrm{H}_{2} \mathrm{O}$. The angle between the two $\mathrm{BF} z$ axes $R$ and $R^{\prime}$ is denoted as $\Delta$. 


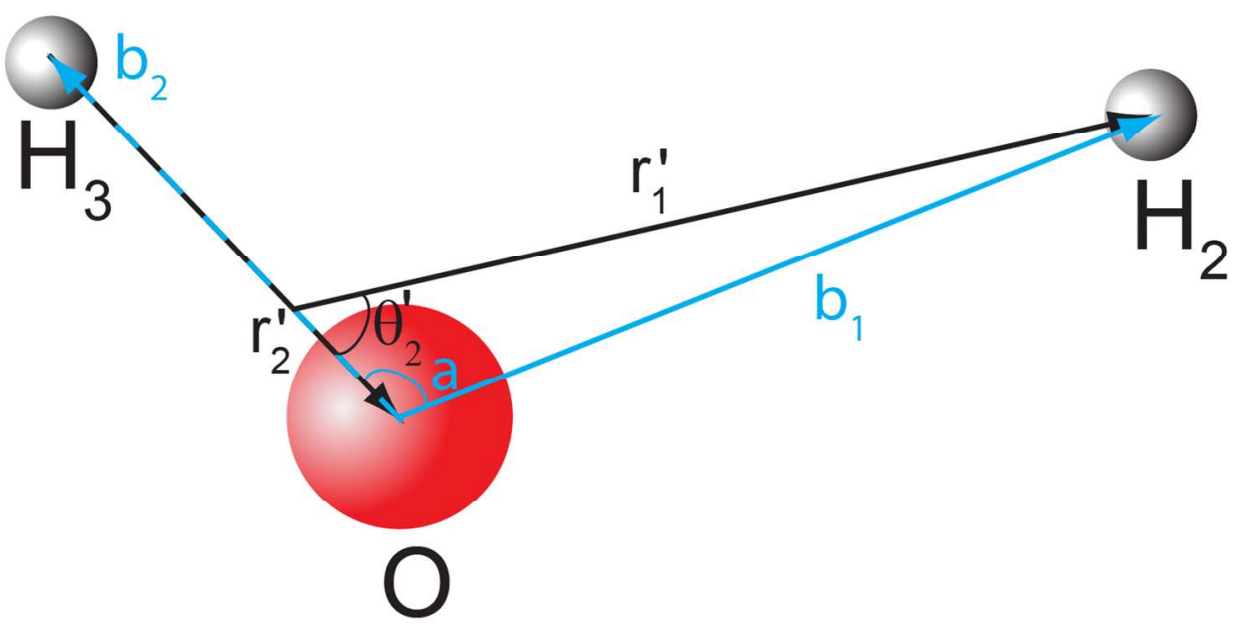

Fig. S2. The Jacobi coordinates $\left(r_{1}^{\prime}, r_{2}^{\prime}, \theta_{2}^{\prime}\right)$ and valence coordinates $\left(b_{1}, b_{2}, a\right)$ of $\mathrm{H}_{2} \mathrm{O}$ product. 


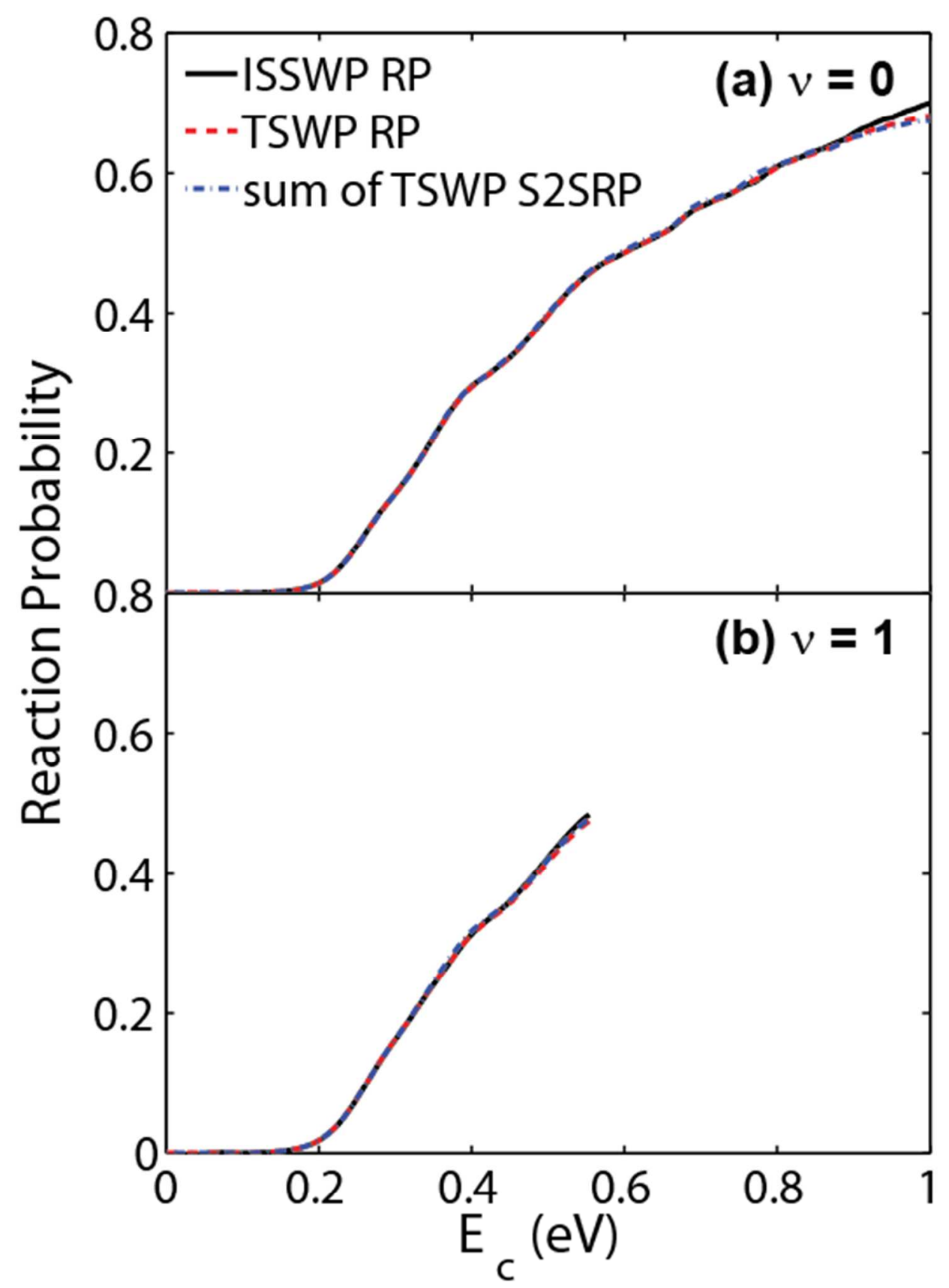

Fig. S3. Initial state-selected total reaction probabilities obtained from three methods: (1) the ISSWP method (black solid), (2) the TSWP method with only reactant propagation (red dashed) and (3) the sum of TSWP state-to-state reaction probabilities over all product states (blue dash dotted). The TSWP calculations used 200 pairs of thermal flux eigenstates. 


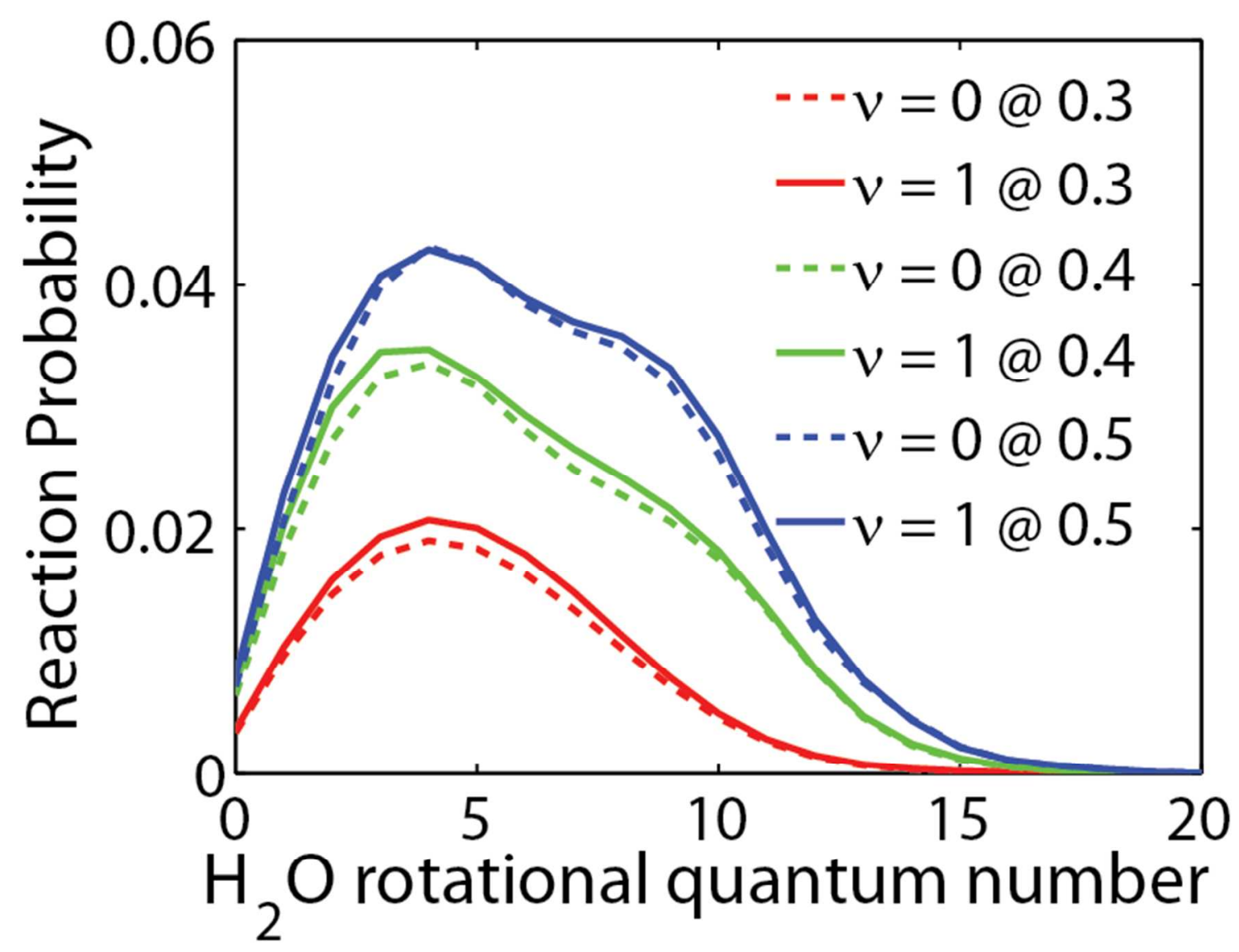

Fig. S4. Rotational state distribution of $\mathrm{H}_{2} \mathrm{O}$ product in the reaction $\mathrm{H}_{2}(\mathrm{v}=0, \mathrm{j}=0)+$ $\mathrm{OH}(\mathrm{v}=0,1, \mathrm{j}=0) \rightarrow \mathrm{H}+\mathrm{H}_{2} \mathrm{O}$ at three different collision energy: $0.3 \mathrm{eV}(\mathrm{red}), 0.4 \mathrm{eV}$ (green) and $0.5 \mathrm{eV}$ (blue) for two vibrational states of $\mathrm{OH}$ reactant: ground (dashed) and first excited (solid) vibrational states. 